\title{
Electric Field Induced Au Nanocrystal Formation in Aqueous Solutions
}

\author{
Jeung Hun Park ${ }^{1,2}$, Mark C. Reuter ${ }^{2}$, Suneel Kodambaka ${ }^{1}$, and Frances M. Ross ${ }^{2}$ \\ ${ }^{1}$ Department of Materials Science and Engineering, University of California Los \\ Angeles, 410 Westwood Plaza, Los Angeles, CA 90095, USA \\ 2 IBM T. J. Watson Research Center, 1101 Kitchawan Road, Yorktown Heights, NY 10598, USA
}

Methods for producing nanoparticles in solution are often developed by exploring a wide range of chemical concentrations and additives until a suitable nanoparticle composition and morphology are obtained [1]. Monitoring the development of crystal structure and morphology during synthesis can help reach the optimized processing parameters more rapidly, reducing the effort and resources required for process development. Recent advances in in situ liquid cell TEM have enabled imaging the particles as they form [2]. This has allowed exploration of the underlying physics of particle crystallization in liquid solutions [3,4]. However, the particle synthesis processes have mainly been influenced by the electron beam, mediated by highly reactive radiolysis species that the beam produces [3-6], and therefore differ from widely used synthesis techniques. Here we discuss a synthesis process that can be observed in the liquid cell, yet is independent of the electron beam. Instead, we use the capability of the liquid cell to apply an electrochemical driving force that then leads to the formation of metal nanoparticles.

In Figure 1, we show a schematic view and three sets of bright-field micrographs taken in a liquid cell [7] equipped with parallel Au electrodes. The images show Au nanoparticles of various sizes distributed in an $\sim 20 \mu \mathrm{m}$ gap between the counter and working electrodes. These nanoparticles were formed by filling the cell with an electrolyte and applying a step-function current, an example of which is shown in Figures 2a and 2b. As can be seen in Figures 1d-1f and Figure 2c, the resulting nanoparticle morphology depends on the liquid thickness. Larger aggregates of $\mathrm{Au}$ form in thinner regions of liquid while isolated particles are produced in thicker liquids.

The electrolytes examined to date, such as $0.5 \mathrm{M} \mathrm{H}_{2} \mathrm{SO}_{4}$ and $0.1 \mathrm{M} \mathrm{ZnSO} / 0.5 \mathrm{M} \mathrm{H}_{2} \mathrm{SO}_{4}$, can be examined for long periods (tens of minutes) without showing particle formation or other beam effects, yet as soon as current flow begins, particles start to form. Note that it is not necessary for Au or other metal ions to be present initially in the electrolyte. This indicates that the source of the metal ions is the counter electrode; current flow through the liquid cell causes Au metal to be oxidized at this electrode, releasing $\mathrm{Au}$ ions into solution. The applied electric field induces ionic diffusion as well as $\mathrm{pH}$ gradients. Changes in $\mathrm{pH}$ can affect the stability of dissolved ions, causing precipitation as metal nanoparticles. The result is the formation of particles when the current/voltage is applied. Beam irradiation is not required; indeed, the images in Figure 1 were recorded during particle formation, but similar particles are observed if we examine the area between electrodes after the current has stopped flowing.

By measuring the local crystallization kinetics - the formation rate, morphology, and composition of these electrogenerated particles as a function of electrolyte composition, electrolyte layer thickness, and spatial configurations around the electrodes - we can obtain insights into their formation. We will compare experimental results obtained in the liquid cell with expectations from a modified diffusion model that includes the diffusion of the ionic species (including radiolytic species, where present), the interactions between species (such as the common-ion effect), the electric field distribution, and the diffusive transport and attachment of $\mathrm{Au}$ at the growing particles. A comparison of theoretical predictions with the experimental observations, particularly for different electric fields, allows us to probe the concentration distribution of the ionic species and the local $\mathrm{pH}$ variations. Pulsing the current 
flow, as shown in Figure 2, enables measurement of the dynamics over which very local (micrometer scale) concentration gradients dissipate by diffusion. The experimental and theoretical approaches presented here may be applicable to measuring more generally such local parameters under an electrochemical stimulus. We will finally discuss the benefits and pitfalls of liquid cell capabilities for the study of crystallization processes.

\section{References:}

[1] B. G. Abdallah, C. Kupitz, P. Fromme, and A. Ros, ACS Nano 7 (2013), 10534 (2013).

[2] N. de Jonge and F. M. Ross, Nature Nanotechnology 6 (2011), 695.

[3] H. Zheng, R. K. Smith, Y.-W. Jun, C. Kisielowski, U. Dahmen, and A. P. Alivisatos, Science 324 (2009), 1309.

[4] J. H. Park, J. M. Grogan, H. H. Bau, S. Kodambaka, and F. M. Ross, Microscopy and Microanalysis 18 Suppl. 2 (2013), 486.

[5] J. H. Park, J. M. Grogan, H. H. Bau, S. Kodambaka, and F. M. Ross, Microscopy and Microanalysis 19 Suppl. 2 (2012), 1098.

[6] J. M. Grogan, N. M. Schneider, F. M. Ross, and H. H. Bau, Nano Lett. 14 (2014), 359.

[7] Hummingbird Sientific Liquid Flow Cell (http://hummingbirdscientific.com/products/liquid-flowholder/), operated in a 300kV FEI CM30 TEM.

[8] We acknowledge the assistance of A. W. Ellis at the IBM T.J. Watson Research Center. We gratefully acknowledge funding from the National Science Foundation (NSF-GOALI: DMR-1310639).

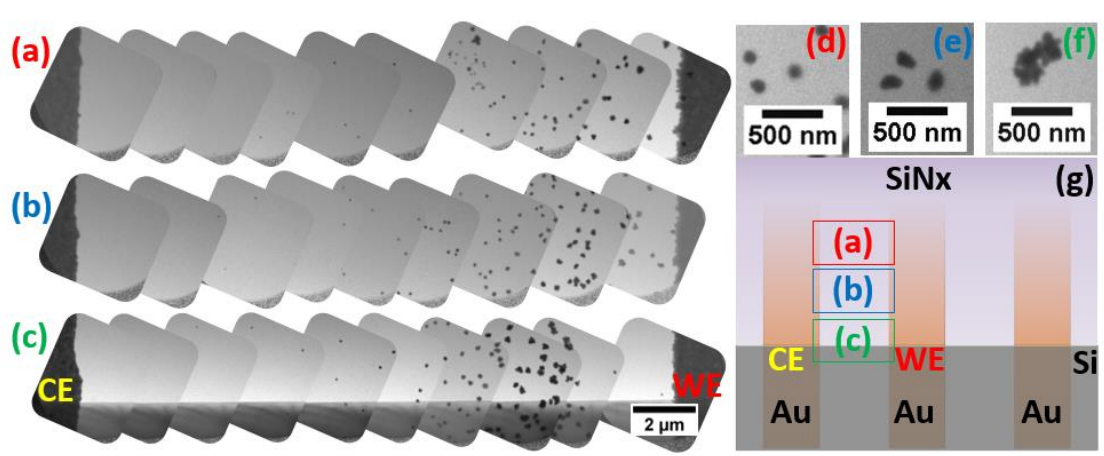

Figure 1. (a-c) Images extracted from a bright field TEM movie obtained during electrochemical growth of $\mathrm{Au}$ nanoparticles across the working electrode (WE) and the counter electrode (CE) in the liquid cell $\mathrm{SiN}_{\mathrm{x}}$ window. The thickness of liquid layer is (a) $1000 \mathrm{~nm}$, (b) $500 \mathrm{~nm}$, and (c) 250 nm. (d-f) Representative morphology of nanoparticles

obtained from (d) $1000 \mathrm{~nm}$, (e) $500 \mathrm{~nm}$, and (f) $250 \mathrm{~nm}$. (g) The layout of the flow-type liquid cell used in this experiment.
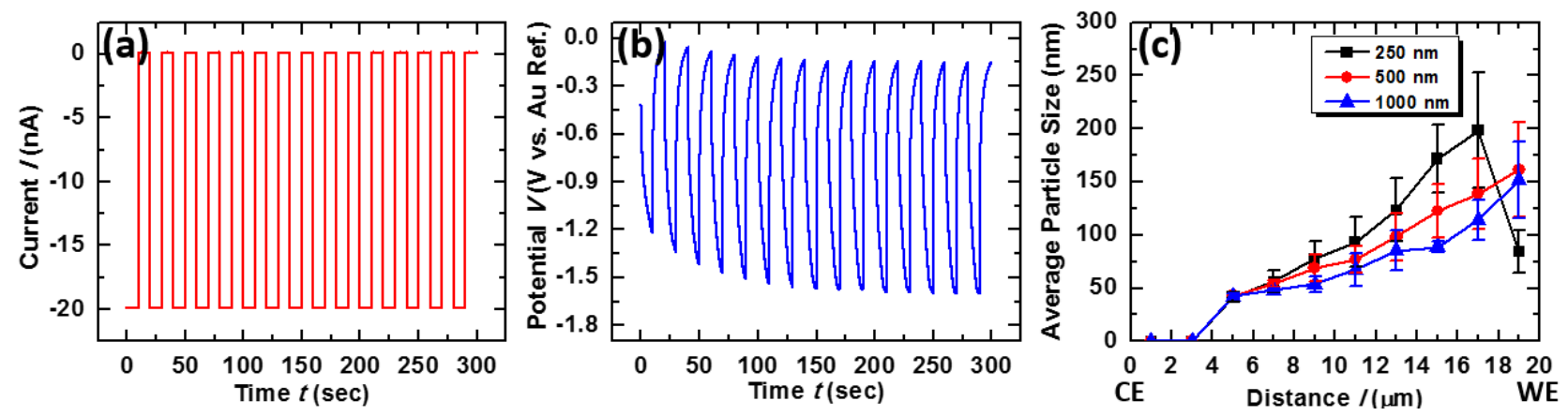

Figure 2. (a) A cyclic chrono-potentiometry of $-20 \mathrm{nA} \times 10 \mathrm{sec}$ and $0 \mathrm{nA} \times 10 \mathrm{sec}$ for a total of 15 cycles. (b) The measured electrochemical potential $V$ vs. time $t$. (c) Average size distribution of $\mathrm{Au}$ particles along a line between the counter and working electrodes. 